\title{
Feasibility of Different Methods after Debonding of Orthodontic Brackets: An In Vivo Study
}

\author{
Juhi Yadav¹, Madhulika Yadav², Danish U. Z. Khan³, Nishi Grover ${ }^{4}$ \\ ${ }^{1}$ Department of Orthodontics, Career Dental College, Lucknow, Uttar Pradesh, India, ${ }^{2}$ Department of Dentistry, \\ Government Medical College, Kannauj, Uttar Pradesh, India, ${ }^{3}$ Department of Dentistry, Era Lucknow Medical \\ College, Lucknow, Uttar Pradesh, India, ${ }^{4}$ Department of Pedodontics, Saraswati Dental College, Lucknow, \\ Uttar Pradesh, India
}

\begin{abstract}
Aim: This study aims to evaluate and compare the discomfort levels during debonding using different three methods and adhesive remnant index (ARI) scores compared. Materials and Methods: Sample comprised 50 female patients from single clinic of an average age of 24 years and 5 months. Three methods used were lift-off debonding instrument (LODI), straight cutter, and Howe plier. At the end of debonding in each quadrant, discomfort levels were assessed using visual analog scale (VAS). Remaining adhesive on enamel surface was studied with the help of ARI. Results: Pain scores were significantly higher for SC method. LODI group showed least discomfort. ARI showed significantly different results with three methods used. Conclusion: Patients showed least discomfort when LODI method was used.
\end{abstract}

Key words: Adhesive remnant index, bonding, debonding, orthodontic brackets, pain

\section{INTRODUCTION}

Fixed orthodontic treatment is no exception in terms of pain experience as other branches of dentistry. Patients report pain during various stages of fixed orthodontic therapy. ${ }^{[1,2]}$ It can be during separator placements, archwire activations, orthopedic forces, and finally during debonding procedures. ${ }^{[3-5]}$ Sometimes due to possibility of pain experiences, patient's willingness to treatment is influenced. ${ }^{[6]}$ Debonding process can be done with the use of special debonding pliers,,$^{[7]}$ ultrasound, ${ }^{[8]}$ laser application, ${ }^{[9]}$ electrothermal debonding, ${ }^{[10]}$ special instruments, and use of special bonding materials presenting thermoexpandable microcapsules to facilitate debonding. Due to pain

\begin{tabular}{|l|l|}
\hline \multicolumn{2}{|c|}{ Access this article online } \\
\hline \multicolumn{1}{|c|}{ Publisher } & \multicolumn{1}{|l|}{ Website: } \\
Www.ijdms.in \\
DOI: 10.30954/IJDMS.1.2019.3
\end{tabular}

perception during debonding, many authors are concerned to study discomfort levels ${ }^{[1]}$ so that pain could be minimized. Ideally, one should use the instrument which can be harmless to the enamel and painless to the patient.

\section{Aim}

The aim of this study was as follows:

1. To compare three debonding methods and the level of discomfort caused by each of them

2. To study which method maintained the integrity of enamel.

\section{MATERIALS AND METHODS}

The sample of 50 female patients was collected from single private clinic and patients had fixed orthodontic appliances removed in both the arches. The sample included patients with Angle Class I malocclusion with permanent teeth and having no restorations. The average age at the time of debonding was 24 years and 5 months. Informed consent forms have been signed by all patients before the start of treatment.

\footnotetext{
Address for Correspondence:

Dr. Juhi Yadav, Department of Orthodontics, Career Dental College, Lucknow, Uttar Pradesh, India. Phone: +91-9415550040.

E-mail: drjuhiyadav@yahoo.com
}

Submission: 29 May 19; Revision: 31 May 19; Acceptance: 27 June 19 
Brackets were $022 \times 028$ Roth prescription [Figure 1] from Class I and were bonded and debonded with the same orthodontist. Before bonding, all necessary precautions were taken and Transbond XT 3M composite [Figure 2] was used in all patients following the manufactures instructions. Patients were instructed that following treatment and during debonding procedure, they need to assess discomfort of the procedure using a Visual analog scale (VAS). This scale comprised millimeter ruler scoring $0-10$.

- $0=$ No pain

- $5=$ Moderate pain

- $10=$ Worst pain (unbearable).

Teeth evaluated were premolar and canine as their brackets were of same size. The debonding methods used were lift-off debonding instrument (LODI) [Figure 3], straight cutter plier (SC) [Figure 4], and Howe plier (HP) [Figure 5]. Remaining adhesive on enamel surface was inspected with electron microscope and adhesive remnant index (ARI) was recorded with the same orthodontist.

- $0=$ No adhesive remaining

- $1=$ Less than half of adhesive remaining

- $2=$ More than half of adhesive remaining

- $3=$ All adhesive remaining.

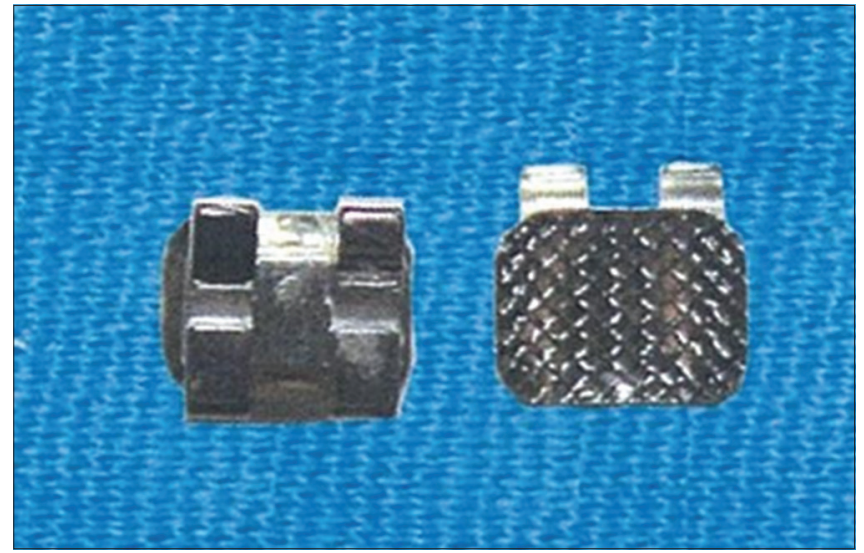

Figure 1: Brackets

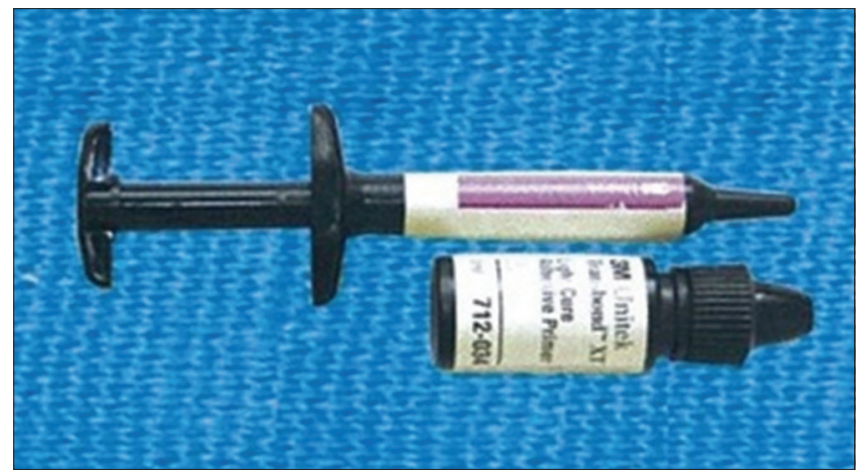

Figure 2: Transbond XT composite

\section{Statistical Analysis}

Continuous data were summarized as mean \pm standard deviation (SD) whereas discrete (categorical) in number $(n)$ and percentage $(\%)$. Continuous groups were compared by one-factor analysis of variance (ANOVA) and the significance of mean difference between (inter) the groups was done by Tukey's honestly significant difference post hoc test after ascertaining normality by Shapiro-Wilks test and homogeneity of variance between groups by Levene's test. Categorical groups were compared by Chi-square $\left(\chi^{2}\right)$ test. A two-tailed $(\alpha=2) P<0.05$ was considered statistically significant. Analyses were performed on SPSS software (Windows version 17.0).

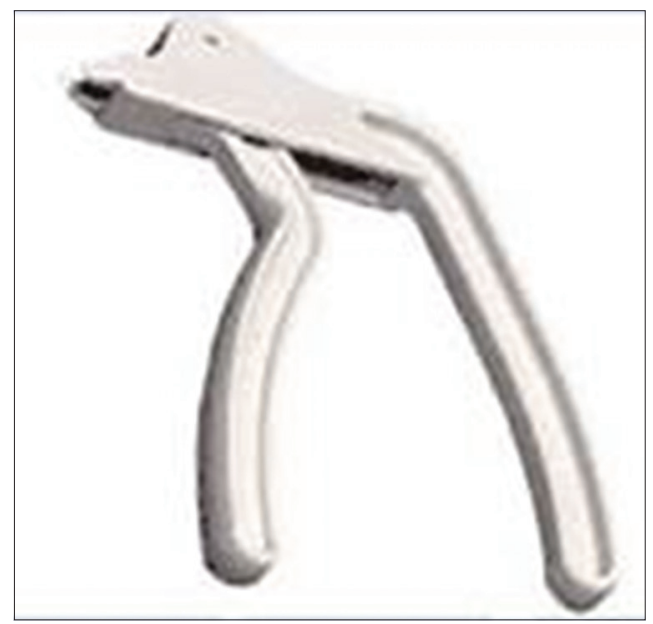

Figure 3: Lift off debonding instrument

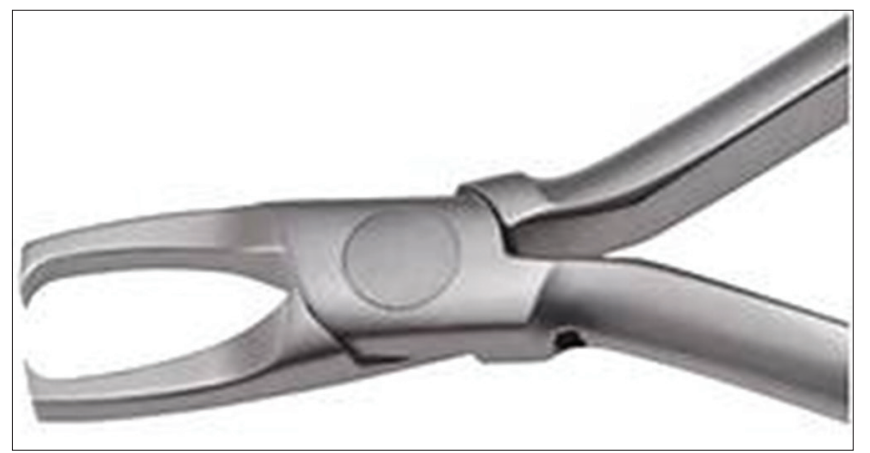

Figure 4: Straight cutter plier

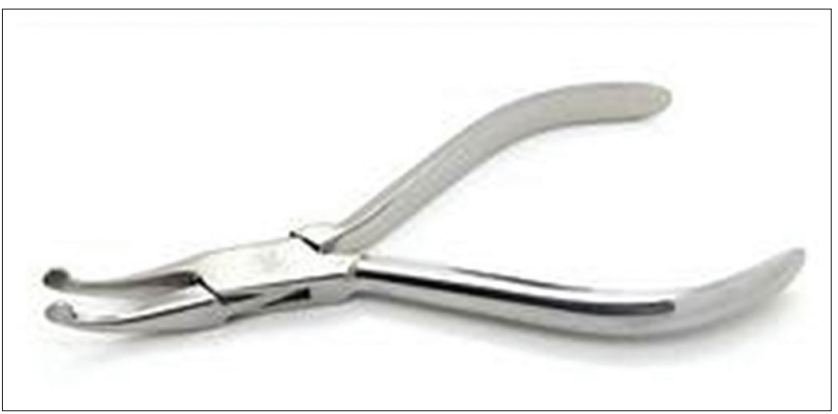

Figure 5: Howe plier 


\section{RESULTS}

The present in vivo study compares the different methods during debonding of orthodontic brackets. A total of 50 samples were recruited and evaluated. The samples were treated (debonded) with LODI, HP, and SC. The primary outcome measures of the study were pain (VAS) and ARI. The objective of the study was to compare the outcome measures between the three groups (LODI, HP, and SC).

\section{Pain}

The pain, i.e., VAS score of three groups is summarized in Table 1. The VAS score of LODI, HP, and SC groups ranged from 2 to 3 , 4 to 5 , and 6 to 8 , respectively, with mean $( \pm \mathrm{SD}) 2.52 \pm 0.50$, $4.46 \pm 0.50$, and $6.90 \pm 0.76$, respectively, and median 3,4 , and 7 , respectively. The mean VAS score was highest in SC followed by HP and LODI, the least SC > HP > LODI.

Comparing the mean VAS score of three groups, ANOVA showed significantly different VAS score among the groups $(F=663.00$, $P<0.001$ ) [Table 1]. Further, comparing the difference in mean VAS score between the groups, Tukey test showed significantly

Table 1: Visual analog scale score of three groups

\begin{tabular}{lccc}
\hline Group & Mean \pm SD $(\boldsymbol{n = 5 0})$ & $\boldsymbol{F}$ & $\boldsymbol{P}$ \\
\hline LODI & $2.52 \pm 0.50$ & 663.00 & $<0.001$ \\
HP & $4.46 \pm 0.50$ & & \\
SC & $6.90 \pm 0.76$ & & \\
\hline
\end{tabular}

VAS score of three groups were summarized in mean \pm SD and compared by ANOVA ( $F$. SD: Standard deviation, LODI: Lift-off debonding instrument, HP: Howe plier, SC: Straight cutter, ANOVA: Analysis of variance
$(P<0.001)$ different and higher VAS score in both HP $(43.5 \%)$ and SC (63.5\%) as compared to LODI [Table 2 and Graph 1]. Furthermore, it was also found significantly $(P<0.001)$ different and higher in SC (35.4\%) as compared to HP.

\section{Ari}

The ARI score of three groups is summarized in Table 3 and also depicted in Graph 2. In LODI method, the ARI score 0 (no adhesive remaining), 1 (less than half of adhesive remaining), 2 (more than half of adhesive remaining), and 3 (all adhesive remaining) were $0(0.0 \%), 13(26.0 \%)$, $31(62.0 \%)$, and $3(12.0 \%)$, respectively. Whereas in HP method, it were $0(0.0 \%), 13(26.0 \%), 37(74.0 \%)$, and $0(0.0 \%)$, respectively, and in SC method, it were $16(32.0 \%)$, $34(68.0 \%), 0(0.0 \%)$, and $0(0.0 \%)$, respectively.

The ARI score, 0 was highest in SC (32.05) and least in both LODI $(0.0 \%)$ and HP (0.0\%). Similarly, ARI score 1 was highest in SC (68.0\%) and least in both LODI (26.0\%) and HP (26.0\%). In contrast, ARI score 2 was highest in HP (74.0\%) followed by LODI $(62.0 \%)$ and least in SC $(0.0 \%)$. Conversely, ARI score 3 was highest in LODI $(12.0 \%)$ and least in both HP $(0.0 \%)$ and SC $(0.0 \%)$.

Comparing the frequency (\%) distribution of ARI score of three groups, Chi-square test showed significantly different frequency of ARI score among the groups (Chisquare $=93.49, P<0.001$ ).

Table 2: Comparison of difference in mean visual analog scale score between groups by Tukey test

\begin{tabular}{lcccc}
\hline Comparison & Mean difference & $\boldsymbol{q}$ & $\boldsymbol{P}$ & $\mathbf{9 5 \%} \mathbf{C l}$ of difference \\
\hline LODI versus HP & -1.94 & 22.76 & $<0.001$ & $1.65-2.23$ \\
LODI versus SC & -4.38 & 51.39 & $<0.001$ & $4.09-4.67$ \\
HP versus SC & -2.44 & 28.63 & $<0.001$ & $2.15-2.73$ \\
\hline
\end{tabular}

q: Tukey test value, Cl: Confidence interval, LODI: Lift-off debonding instrument, HP: Howe plier, SC: Straight cutter

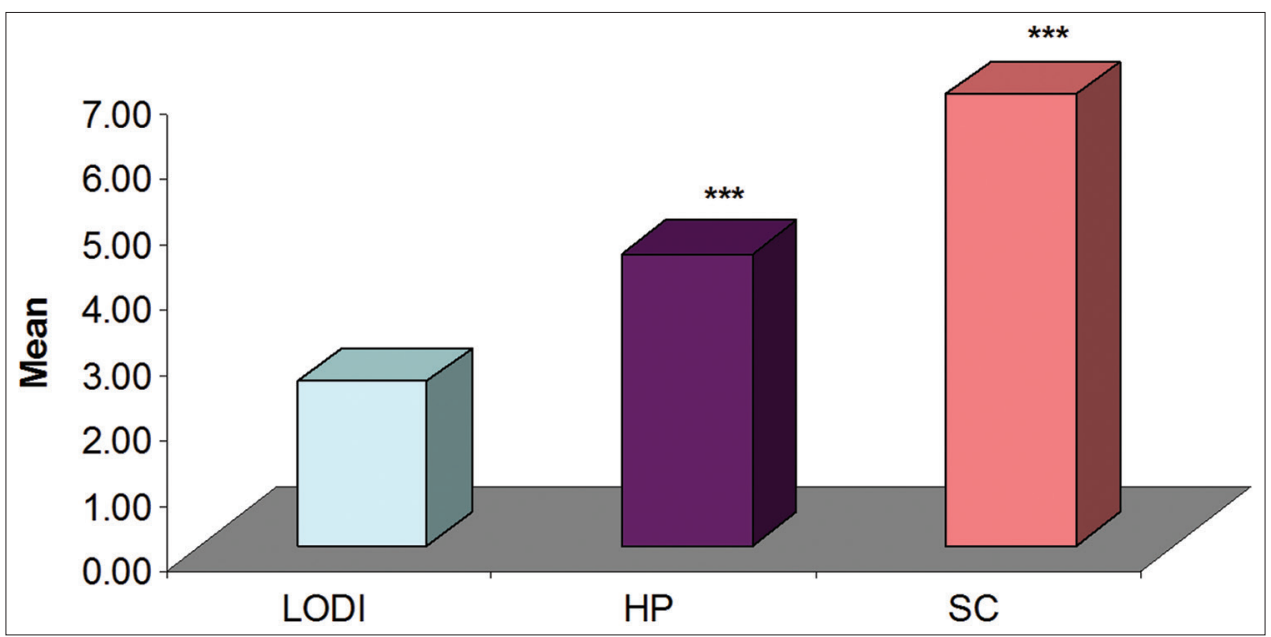

Graph 1: Mean VAS score of three groups. ${ }^{* *} P<0.001$ as compared to lift-off debonding instrument 


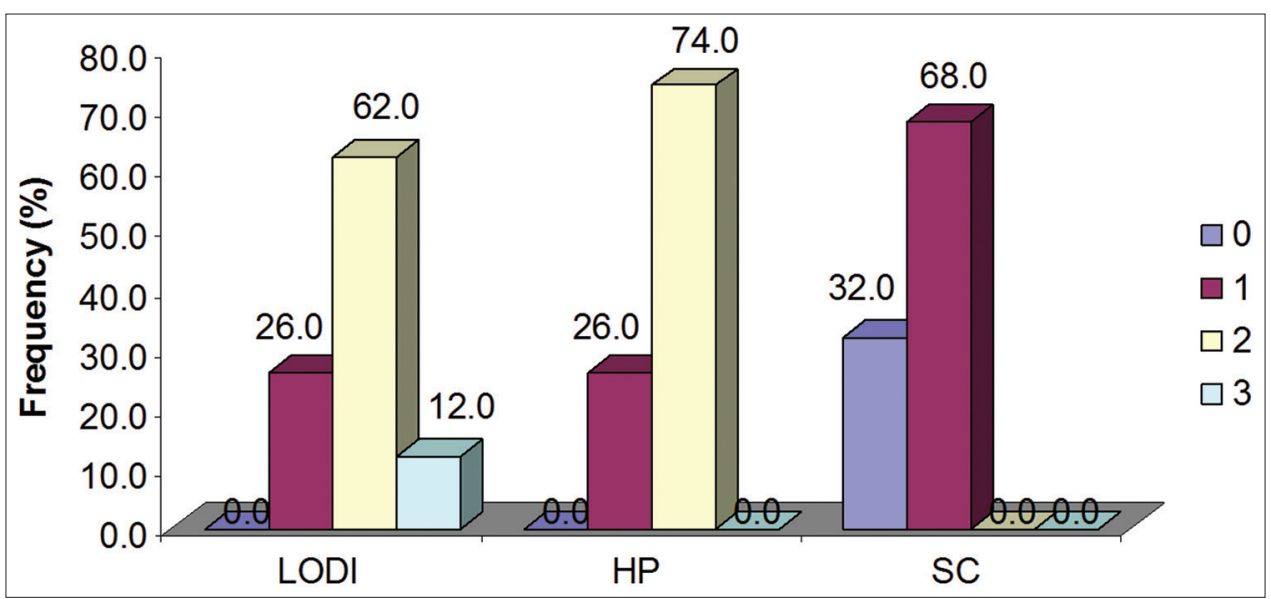

Graph 2: Distribution of adhesive remnant index score of three groups. 0: No adhesive remaining, 1: Less than half of adhesive remaining, 2: More than half of adhesive remaining, and 3: All adhesive remaining

Table 3: Adhesive remnant index score of three groups $(n=50)$

\begin{tabular}{lccccc}
\hline $\begin{array}{l}\text { ARI } \\
\text { score }\end{array}$ & $\begin{array}{c}\text { LODI, } \\
\boldsymbol{n}(\%)\end{array}$ & $\begin{array}{c}\text { HP, } \\
\boldsymbol{n}(\%)\end{array}$ & $\begin{array}{c}\text { SC, } \\
\boldsymbol{n}(\%)\end{array}$ & $\chi^{2}$ & $\boldsymbol{P}$ \\
\hline 0 & $0(0.0)$ & $0(0.0)$ & $16(32.0)$ & 93.49 & $<0.001$ \\
1 & $13(26.0)$ & $13(26.0)$ & $34(68.0)$ & & \\
2 & $31(62.0)$ & $37(74.0)$ & $0(0.0)$ & & \\
3 & $6(12.0)$ & $0(0.0)$ & $0(0.0)$ & & \\
\hline
\end{tabular}

0 : No adhesive remaining, 1: Less than half of adhesive remaining, 2: More than half of adhesive remaining, 3 : All adhesive remaining. The ARI score of three groups was summarized in $(n)$ and $(\%)$ and compared by Chi-square test. ARI: Adhesive remnant index, LODI: Lift-off debonding instrument, HP: Howe plier, SC: Straight cutter

\section{DISCUSSION}

Science is that branch in which we continuously strive for betterment each day. Hence, medical and dental field is no exception. We are always striving to make patients treatment painless and harmless with least of side effects. ${ }^{[12,13]}$ In the field of orthodontics, debonding causes discomfort and pain in patients and the intensity of pain varies with each individual. Hence, as an orthodontist, we try to find the method which is least painful at debonding stage. Hence, the purpose of the study became to evaluate the best method among the methods which are commonly used for debonding ${ }^{[14,15]}$ determining patients discomfort and amount of adhesive remaining on the enamel surface which, in turn, affects potential enamel damage.

The results of this study tell that LODI method caused least discomfort ${ }^{[16]}$ and SC method the maximum. These results are same as earlier studies. ${ }^{[17,18]}$ According to Williams and Bishara, ${ }^{[11]}$ the direction of debonding force can influence the discomfort level of patient. Patients can bear intrusive forces more than other directional forces. The direction of force has no control with the method used so results can vary with the patients. Hence, to derive conclusions, we need instruments which all direct forces in single direction and with same magnitude. BRP, SC, and HP exert forces of similar magnitude, but LODI requires lower and more constant force levels.

Bishara quoted $1000 \mathrm{~g}$ of force as appropriate which can be directly applied to tooth surface. Anterior and posterior arches have different threshold for force levels so to rule out any discrepancy we choose canine and premolar ${ }^{[19]}$ brackets as they are of same dimensions. As different studies quote incisor debonding to be most painful, because tactile sensory threshold is about $\mathrm{lg}$ for anterior part of dentition. In posterior section, it is about $5-10 \mathrm{~g}$. Hence, using canine and premolar brackets, we tried to standardize the study effectively. Different studies suggested the threshold difference of pain in between males and females. Hence, to rule out the discrepancy, we have taken only females for the study. We tried to minimize the variables trying to affect the study results. During comparing the three methods, we compared the effect of debonding on enamel surface also. Artun and Bergland ${ }^{[20]}$ suggested that enamel will be protected if adhesive line of fracture lies within adhesive layer instead of enamel and adhesive interface. ARI gives a very good perspective to study which method maintained the integrity of enamel most. A portable electron microscope is used to evaluate ARI scores. The results showed a significant difference among three debonding procedures. ARI score for SC method was smaller as compared to LODI and HP methods and SC method indicated greater potential for enamel damage ${ }^{[17]}$ as least amount of adhesive was left during debonding and generates more forces between enamel and adhesive layer. This presents higher risk to injure enamel surface. Hence, SC method is not recommended method for debonding as suggested by other studies also, ${ }^{[21,22]}$ as it causes more patient discomfort and greater potential for enamel damage. Few studies also quoted that brackets debonded with LODI method remain structurally intact and reusable, whereas $\mathrm{SC}$ method causes structural deformation of brackets. 


\section{CONCLUSION}

The present study found LODI the best method for debonding after the fixed orthodontic treatment as it creates least pain and more adhesive on enamel surface which helps avoiding damage to enamel surface. SC method proves to be least desirable as it creates lots of pain and more prone to enamel damage. However, findings of this study may need further validation on larger sample size and comparisons with other methods.

\section{REFERENCES}

1. d'Ornellas Pereira JC Jr., Weissheimer A, de Menezes LM, de Lima EM, Mezomo M. Change in the pulp chamber temperature with different stripping techniques. Prog Orthod 2014;15:55.

2. Sobouti F, Rakhshan V, Chiniforush N, Khatami M. Effects of laser-assisted cosmetic smile lift gingivectomy on postoperative bleeding and pain in fixed orthodontic patients: A controlled clinical trial. Prog Orthod 2014;15:66.

3. Loewenstein WR, Rathkamp R. A study on the pressoreceptive sensibility of the tooth. J Dent Res 1955;34:287-94.

4. Krishnan V. Orthodontic pain: From causes to management - a review. Eur J Orthod 2007;29:170-9.

5. Takla PM, Shivapuja PK. Pulpal response in electrothermal debonding. Am J Orthod Dentofacial Orthop 1995;108:623-9.

6. Haynes S. Discontinuation of orthodontic treatment relative to patient age. J Dent 1974;2:138-42.

7. Brown DF, Moerenhout RG. The pain experience and psychological adjustment to orthodontic treatment of preadolescents, adolescents, and adults. Am J Orthod Dentofacial Orthop 1991;100:349-56.

8. Kluemper GT, Hiser DG, Rayens MK, Jay MJ. Efficacy of a wax containing benzocaine in the relief of oral mucosal pain caused by orthodontic appliances. Am J Orthod Dentofacial Orthop 2002;122:359-65.

9. Lee-Knight CT, Wylie SG, Major PW, Glover KE, Grace M. Mechanical and electrothermal debonding: Effect on ceramic veneers and dental pulp. Am J Orthod Dentofacial Orthop 1997;112:263-70.

10. Boyer DB, Engelhardt G, Bishara SE. Debonding orthodontic ceramic brackets by ultrasonic instrumentation. Am J Orthod Dentofacial Orthop 1995;108:262-6.
11. Williams OL, Bishara SE. Patient discomfort levels at the time of debonding: A pilot study. Am J Orthod Dentofacial Orthop 1992;101:313-7.

12. Farzanegan F, Zebarjad SM, Alizadeh S, Ahrari F. Pain reduction after initial archwire placement in orthodontic patients: A randomized clinical trial. Am J Orthod Dentofacial Orthop 2012;141:169-73.

13. Kokich VG. Do you have an evidence-based practice? Am J Orthod Dentofacial Orthop 2013;143:1.

14. Parrish BC, Katona TR, Isikbay SC, Stewart KT, Kula KS. The effects of application time of a self-etching primer and debonding methods on bracket bond strength. Angle Orthod 2012;82:131-6.

15. Cehreli SB, Polat-Ozsoy O, Sar C, Cubukcu HE, Cehreli ZC. A comparative study of qualitative and quantitative methods for the assessment of adhesive remnant after bracket debonding. Eur J Orthod 2012;34:188-92.

16. Elekdag-Turk S, Isci D, Ozkalayci N, Turk T. Debonding characteristics of a polymer mesh base ceramic bracket bonded with two different conditioning methods. Eur J Orthod 2009;31:84-9.

17. Bennett CG, Shen C, Waldron JM. The effects of debonding on the enamel surface. J Clin Orthod 1984;18:330-4.

18. Kinch AP, Taylor H, Warltier R, Oliver RG, Newcombe RG. A clinical study of amount of adhesive remaining on enamel after debonding, comparing etch times of 15 and 60 seconds. Am J Orthod Dentofacial Orthop 1989;95:415-21.

19. Manly RS, Pfaffman C, Lathrop DD, Keyser J. Oral sensory thresholds of persons with natural and artificial dentitions. J Dent Res 1952;31:305-12.

20. Artun J, Bergland S. Clinical trials with crystal growth conditioning as an alternative to acid-etch enamel pretreatment. Am J Orthod 1984;85:333-40.

21. Knösel M, Mattysek S, Jung K, Kubein-Meesenburg D, SadatKhonsari R, Ziebolz D. Suitability of orthodontic brackets for rebonding and reworking following removal by air pressure pulses and conventional debracketing techniques. Angle Orthod 2010;80:461-7.

22. Knösel M, Mattysek S, Jung K, Sadat-Khonsari R, KubeinMeesenburg D, Bauss $\mathrm{O}$, et al. Impulse debracketing compared to conventional debonding. Angle Orthod 2010;80:1036-44.

How to cite this article: Yadav J, Yadav M, Khan DUZ, Grover N. Feasibility of Different Methods after Debonding of Orthodontic Brackets: An In Vivo Study. Int J Dent Med Spec 2019;6(1):4-8.

Source of Support: None; Conflict of Interest: None 